\section{Vol. 32, Issue 10, October 2009}

\section{Editorials}

547 The Influence of Sex in Non-Small Cell Lung Cancer

Wheatley-Price, P. (Ottawa); Blackhall, F.; Thatcher, N. (Manchester)

550 Microenvironmental Influences and Antigenic Stimulation in Chronic Lymphocytic Leukemia Seiler, T.; Hiddemann, W. (München)

552 Beyond Platinum for Metastatic and Recurrent Carcinoma of the Cervix Tewari, K.S.; Monk, B.J. (Orange, CA)

\section{Original Articles}

556 Detection and Prediction of Breast Cancer Using Double Phase Tc-99m MIBI Scintimammography in Comparison with MRI

Kim, I.J.; Kim, Y.-K.; Kim, S.-J. (Busan)

562 UFT plus Oral Folinic Acid with Alternating Oral and Intravenous Vinorelbine in Patients with Metastatic Breast Cancer Previously Treated with Anthracyclines and Taxanes

Coşkun, U.; Kaya, A.O.; Alkıs, N.; Buyukberber, S.; Alııı, S.; Celenkoglu, G.; Uncu, D. (Ankara); Özkan, M. (Kayseri); Sevinc, A. (Gaziantep); Benekli, M. (Ankara), for the Anatolian Society of Medical Oncology

569 Lung Cancer in the Canton of St. Gallen, Eastern Switzerland: Sex-Associated Differences in Smoking Habits, Disease Presentation and Survival Cerny, D.; Cerny, T.; Ess, S.; D`Addario, G.; Früh, M. (St. Gallen)

574 The Relationship between Cyclooxygenase-2, CD44v6, and nm23H1 in Esophageal Squamous Cell Carcinoma Liu, W.K. (Xi'an); Fu, Q. (Zheng Zhou); Li, Y.M. (Chengdu); Jiang, X.Y.; Zhang, M.P.; Zhang, Z.X. (Xi’an)

Clinical Cases

580 Toxic Epidermal Necrolysis Related to Pemetrexed and Carboplatin with Vitamin B12 and Folic Acid Supplementation for Advanced Non-Small Cell Lung Cancer

Bosch-Barrera, J.; Gaztañaga, M.; Ceballos, J.; Pérez-Gracia, J.L.; López-Picazo, J.M.; García-Foncillas, J.; Ferrer, M.; Sanz, M.L.; Pretel, M.; Idoate, M.A.; Gil-Bazo, I. (Pamplona)
Band 32, Heft 10, Oktober 2009

Editorials

547 Geschlechtspezifische Unterschiede beim nicht-kleinzelligen Bronchialkarzinom Wheatley-Price, P. (Ottawa); Blackhall, F.; Thatcher, N. (Manchester)

550 Einflüsse des Microenvironment und Antigenstimulation bei chronisch-lymphatischer Leukämie Seiler, T.; Hiddemann, W. (München)

552 Behandlung des metastasierten und rezidivierten Zervixkarzinoms jenseits von Platin Tewari, K.S.; Monk, B.J. (Orange, CA)

Originalarbeiten

556 Detektion und Prädiktion von Mammakarzinomen mit Zweiphasen-Tc-99m-MIBI-Szintimammographie im Vergleich mit MRI

Kim, I.J.; Kim, Y.-K.; Kim, S.-J. (Busan)

562 UFT und orale Folinsäure mit alternierend oralem und intravenösem Vinorelbin bei mit Anthrazyklinen und Taxanen vorbehandelten Patientinnen mit metastasiertem Brustkrebs

Coşkun, U.; Kaya, A.O.; Alkıs, N.; Buyukberber, S.; Alıcı, S.; Celenkoglu, G.; Uncu, D. (Ankara); Özkan, M. (Kayseri); Sevinc, A. (Gaziantep); Benekli, M. (Ankara), for the Anatolian Society of Medical Oncology

569 Lungenkrebs im Kanton St. Gallen, Ost-Schweiz: Eine retrospektive Analyse der geschlechtsspezifischen Unterschiede bezüglich Rauchverhalten, Krankheitspräsentation und Überleben Cerny, D.; Cerny, T.; Ess, S.; D`Addario, G.; Früh, M. (St. Gallen)

574 Das Verhältnis zwischen Cyclooxygenase-2, CD44v6 und nm23H1 beim Plattenepithelkarzinom des Ösophagus Liu, W.K. (Xi'an); Fu, Q. (Zheng Zhou); Li, Y.M. (Chengdu); Jiang, X.Y.; Zhang, M.P.; Zhang, Z.X. (Xi'an)

Kasuistiken

580 Toxische epidermale Nekrolyse im Zusammenhang mit Pemetrexed und Carbonplatin mit Vitamin B12 und Folsäure zur Behandlung des forgeschrittenen nicht-kleinzelligen Bronchialkarzinoms Bosch-Barrera, J.; Gaztañaga, M.; Ceballos, J.; Pérez-Gracia, J.L.; López-Picazo, J.M.; García-Foncillas, J.; Ferrer, M.; Sanz, M.L.; Pretel, M.; Idoate, M.A.; Gil-Bazo, I. (Pamplona)

\section{KARGER}

Fax +497614520714

Information@Karger.de

www.karger.com

\section{(C) 2009 S. Karger GmbH, Freiburg}

Artikel (Volltext) und Inhaltsverzeichnisse

sowie das vorläufige Inhaltsverzeichnis des nächsten Heftes: www.karger.com/onk_bk.htm 


\section{Vol. 32, Issue 10, October 2009}

586 Primary Manifestation of Small Lymphocytic Lymphoma in the Prostate

Fehr, M. (Chur); Templeton, A.; Cogliatti, S. (St. Gallen); Aebersold, F.; Egli, F.; Gillessen, S.; Cathomas, R. (Chur)

591 Rectal Intussusception due to Re-Recurrent Ovarian Fibrosarcoma

Langner, I.; Glitsch, A.; Krüger, W.; Vogelgesang, S.; Langner, S.; von Bernstorff, W.; Heidecke, C.D. (Greifswald)

595 Complete Remission of Metastatic and Relapsed Uterine Cervical Cancers Using Weekly Administration of Bevacizumab and Paclitaxel/ Carboplatin

Takano, M.; Kikuchi, Y. (Tokorozawa); Kita, T. (Tokyo); Goto, T. (Hidaka); Yoshikawa, T.; Kato, M.; Watanabe, A.; Sasaki, N.; Miyamoto, M. (Tokorozawa); Inoue, H.; Ohbayashi, M. (Kamakura)

\section{Review Articles}

599 Incidence of Febrile Neutropenia and Myelotoxicity of Chemotherapy: A Meta-Analysis of Biosimilar G-CSF Studies in Breast Cancer, Lung Cancer, and Non-Hodgkin's Lymphoma

Engert, A. (Köln); del Giglio, A. (Sao Paolo); Bias, P. (Ulm); Lubenau, H. (Mannheim); Gatzemeier, U.; Heigener, D. (Großhansdorf)

605 Cancer Stem Cells in Solid Tumors $\mathrm{Er}, \mathrm{O}$. (Istanbul)

611 Book Review

612 PharmaNews / PharmaTicker

614 Meetings and Conferences

598 Imprint

\section{Band 32, Heft 10, Oktober 2009}

586 Kleinzelliges lymphozytisches Lymphom Erstmanifestation als Prostatainfiltration Fehr, M. (Chur); Templeton, A.; Cogliatti, S. (St. Gallen); Aebersold, F.; Egli, F.; Gillessen, S.; Cathomas, R. (Chur)

591 Rektale Invagination durch ein Zweitrezidiv eines ovariellen Fibrosarkoms

Langner, I.; Glitsch, A.; Krüger, W.; Vogelgesang, S.; Langner, S.; von Bernstorff, W.; Heidecke, C.D. (Greifswald)

595 Vollständige Remission bei metastatischem und wiederkehrendem Gebärmutterhalskrebs nach wöchentlicher Gabe von Bevacizumab und Paclitaxel/Carboplatin

Takano, M.; Kikuchi, Y. (Tokorozawa); Kita, T. (Tokyo); Goto, T. (Hidaka); Yoshikawa, T.; Kato, M.; Watanabe, A.; Sasaki, N.; Miyamoto, M. (Tokorozawa); Inoue, H.; Ohbayashi, M. (Kamakura)

\section{Übersichtsarbeiten}

599 Inzidenz der febrilen Neutropenie und Myelotoxizität der Chemotherapie: Meta-Analyse von Biosimilar-G-CSF-Studien bei Brustkrebs, Bronchialkarzinom und Non-Hodgkin-Lymphom Engert, A. (Köln); del Giglio, A. (Sao Paolo); Bias, P. (Ulm); Lubenau, H. (Mannheim); Gatzemeier, U.; Heigener, D. (Großhansdorf)

605 Krebsstammzellen bei soliden Tumoren Er, O. (Istanbul)

611 Buchbesprechung

612 PharmaNews / PharmaTicker

614 Tagungen und Kongresse

598 Impressum
Forthcoming papers are listed on page 616.
Einen Ausblick auf den Inhalt der kommenden Hefte finden Sie auf Seite 616.

\section{KARGER}

Fax +497614520714

Information@Karger.de

www.karger.com

\section{(C) 2009 S. Karger GmbH, Freiburg}

